\title{
Milling Simulation with an adaptive Strategy
}

\author{
Klaus Weinert (weinert@isf.mb.uni-dortmund.de) \\ Department of Machining Technology, University of Dortmund, Germany \\ Frank Albersmann, Georg Guntermann \\ Department of Machining Technology, University of Dortmund, Germany
}

Key words: 3-axis NC machining, sculptured-surface machining, adaptive milling simulation

Abstract: When milling sculptured surfaces the major problems are unsatisfactory process reliability and the limited precision due to milling-cutter deflections caused by the cutting force. One method for improving the process reliability and the surface quality is the computer-based engagement analysis and feed-rate adaption. The aim of the feed-rate adaption is to avoid intolerably high tool loads as they occur while semi-finishing sculptured surfaces. The use of an adaptive simulation system can ensure an optimal agreement between the simulation model and the real cutting process.

\section{INTRODUCTION}

As form tools are necessary for a wide range of production technologies productivity can be greatly improved and costs can be decreased (e.g. drop-forging and injection-molding) by using more economical and more flexible methods to manufacture such molds. One of these ways is direct HSC-milling of hardened steel, which is becoming more and more popular in the die-and-mold manufacturing industry [2]. This technology has been developed to practical usability, actively supported by research efforts in the areas of machine tools, controls and tools.

There are two remaining major problems concerning this cutting process: The poor process reliability -compared to erosive technologies - due to changing engagement conditions especially while semi-finishing and the problem of contour deviations caused by cutting-tool deformations due to cutting forces.

Process reliability is considerably influenced by the risk of tool breakage. The most important reason for tool breakage is that cutting forces increase significantly due to varying real offset conditions.

Contour-faults are caused by two major factors: the limited dynamic capability of the HSCmilling-machine and the deformation of the cutting tool. The first of these influences can be reduced by continuous aligned milling paths and intelligent control-algorithms like feedforward or look-ahead. Cutter deformation, the second factor having an influence on contour faults is caused by changing cutting forces due to changing engagement conditions and local surface-overmeasures.

These problems have to be minimized in order to take full advantage of the greater productivity of the HSC-milling process.

There are different ways for improving the process reliability:

- Milling in discrete steps with reduced surface-overmeasure in order to reduce cuttingforces,

- milling with an overall reduced feed-rate in oder to reduce peaks of the cutting forces, escpecially in areas of locally increased overmeasure,

- manual feed-rate adaption by the worker during the process,

- computer-based engagement analysis and feed-rate adaption.

The first and the second methods can be implemented without problems. The disadvantage of these methods is a significant increase in machining time. The efficiency of the third

The original version of this chapter was revised: The copyright line was incorrect. This has been corrected. The Erratum to this chapter is available at DOI: 10.1007/978-0-387-35392-0_40 
method, manual feed-rate adaption, depends on the skill and the attention of the worker. The high feed-rates of modern HSC-milling machines make it almost impossible for the worker to react fast enough to changing offset conditions.

A method for ensuring high process reliability while decreasing contour-faults is the feedrate adjustment based on the calculation of engagement conditions and cutting volumes. This paper describes a milling simulation developed to implement this method and thus gives us an insight into the simulation kernel. The concept of an adaptive simulation system is explained and the efficiency of the approach developed is demonstrated with a practical example.

\section{PROCESS RELIABILITY IMPROVEMENTS BY SIMULATION AND FEED-RATE ADAPTION}

As shown in several papers, accuracy, process reliability and the time required for the milling process can be improved significantly when using different cutting process models to determine the cutting conditions $[1,7,13]$. The crucial aspect is that all models require knowledge of the cutting conditions. This is especially difficult when milling sculptured surfaces, which occur almost exclusively in the die-and-mold manufacturing industry. For 2 and $2 \frac{1}{2}$ dimensional problems, the engagement conditions can be determined from the NC paths themselves and are constant over a wide range. When milling sculptured surfaces, the engagement conditions constantly vary at a very quick rate and, in general, it is impossible to calculate these values from the $\mathrm{NC}$ paths.

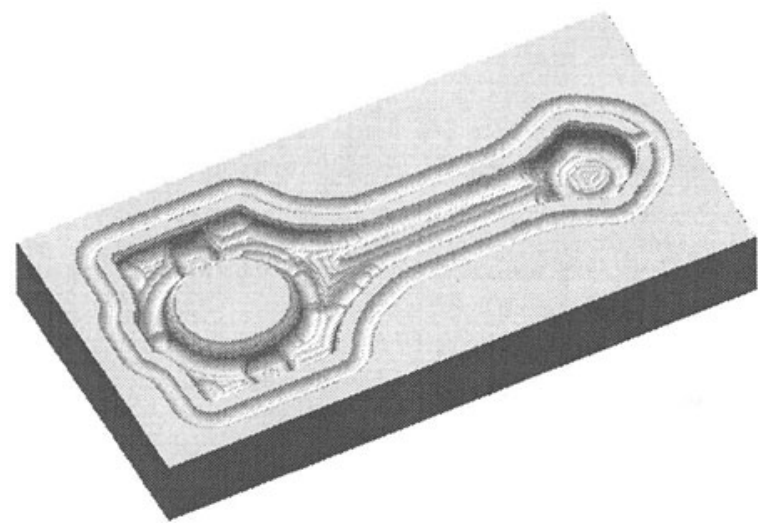

Figure 1. Rendered image of the resulting surface calculated by the milling simulation of the rough milling (5301 paths, $8 \mathrm{~s}$ computing time on a Pentium PC, $133 \mathrm{MHz}$ ).

The development of an efficient 3-axis, milling process simulation makes it possible to calculate the actual geometry of the machined part at any given time during the processing of the NC paths and to verify the automatically generated NC paths [8]. The simulation is based on a discrete volume model and our method diminishes the time and memory space the computer requires for the simulation process, an aspect which has been neglected in the literature up to now. Details of the simulation kernel are described in section 3. An example is given in figure 1. The resulting surface of the workpiece as calculated by the milling simulation of the NC paths for rough-milling is displayed as a rendered image. The computing time necessary to calculate this result for $5301 \mathrm{NC}$ paths is 8 seconds on a Pentium PC. 
But what makes the simulation system suitable for feed-rate adaption is that the simulation allows us to calculate the engagement conditions during the milling of sculptured surfaces. This analysis is necessary to determine the exact cutting-chip volume and afterwards the resulting cross-sectional area of the chip as a function of the pressure-angle $\varphi$ for all positions of the NC-data (Figure 2).

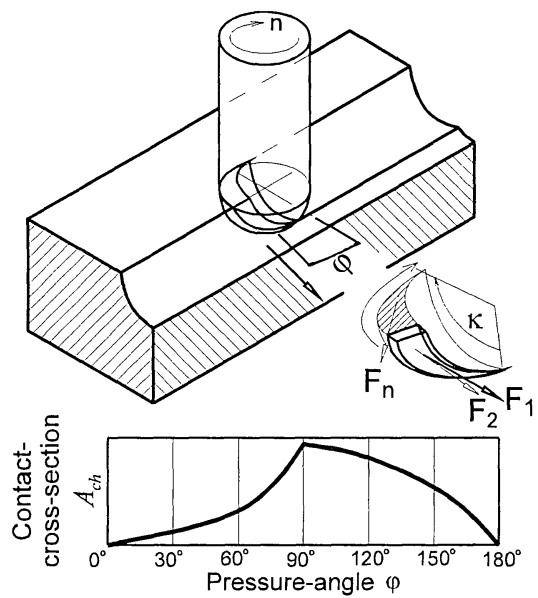

Figure 2. Analysis of engagement conditions

\section{2..1 Basic method of feed-rate adaption}

In figure 3 the main idea of the feed-rate adaption is shown in a graph. The calculations are based on the cross-sectional area of cut $A_{c h}$, derived from the analysis of engagement conditions, described above. With respect to feed-rate adaption this factor is modified by two correction factors, in order to take into account the technological effects of cutting symmetry and path inclination. These factors are used to virtually increase or decrease the real crosssectional area of cut. The modified cross-sectional area is calculated by the formula:

$$
A_{\text {mod }}=A_{c h} \cdot \delta_{G e o} \cdot \delta_{c l}
$$

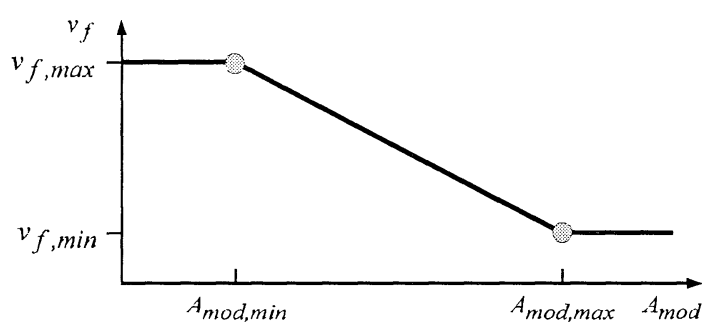

Figure 3. Relation between cross-sectional area and feed-rate

If the calculated cross-sectional area $A_{\text {mod }}$ is below a specified value, the maximum feed-rate is used. If $A_{\text {mod }}$ lies between the maximum and minimum values $\left(A_{\text {mod,min }}\right.$ and $\left.A_{\text {mod,max }}\right)$, the actual feed-rate is decreased linearly: 


$$
v_{f}=v_{f, \text { max }}-\frac{\left(v_{f, \text { max }}-v_{f, \text { min }}\right)}{\left(A_{\text {mod,max }}-A_{\text {mod,min }}\right)} \cdot\left(A_{\text {mod }}-A_{\text {mod,min }}\right)
$$

If the modified value $A_{m o d}$ is higher than $A_{\text {mod,max }}$, the feed-rate will not be further reduced, i.e. it remains on the lowest possible level defined by $v_{f, m i n}$. If this occurs, a warning message is generated, indicating that the maximum cross-sectional area of cut has been exceeded.

\section{2..2 Symmetry of engagement}

To take the technological influences of cutting symmetry and path inclination into account two correction factors are defined. The first correction factor $\delta_{G e o}$ describes the influence of symmetry of engagement (Figure 4 ). First a symmetry factor $f_{\text {sym }}$ is calculated, defining the ratio between cross-sectional area of cut on the left side (viewed in feed-direction) and on the right side. This factor describes the engagement situation: whether it is a symmetrical engagement at the tool tip or only at the circumference of the cutter. The symmetry factor is defined as:

$$
f_{s y m}=\frac{\min \left(A_{c h, l e}, A_{c h, r i}\right)}{\max \left(A_{c h, l e}, A_{c h, r i}\right)}
$$

If there is a complete symmetrical engagement, the factor $\delta_{G e o}$ is set at 1 (figure 4 ). The factor is increased continuously to the maximum $\delta_{G e o, \max }$, for symmetry values between 1 and a definable minimum value $f_{\text {sym,min }}$ :

$$
\delta_{G e o}=\delta_{G e o, \max }-\frac{\left(\delta_{G e o, \max }-1\right) \cdot\left(f_{s y m}-f_{s y m, \min }\right)}{\left(1-f_{s y m, \min }\right)}
$$

The reason for this behaviour is that an engagement only at the circumference of the cutter leads to higher cutter deflections in comparison with a symmetrical engagement in the area of the tool tip.

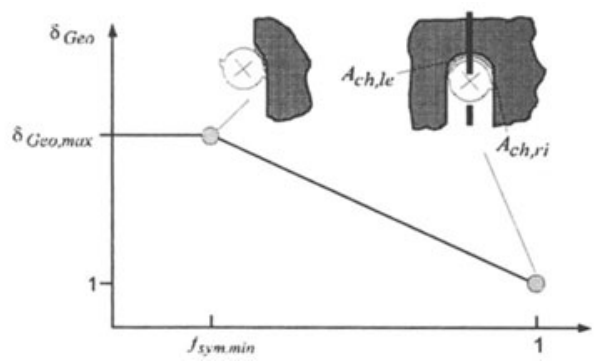

Figure 4. Relation between cross-sectional area and feed-rate

\section{2..3 Influence of milling direction}

It is a technological fact, that the tool load for downward directed cutting paths (plunge cutting) is noticeably higher than in the case of an upward directed path. To take this into account a correction factor for the path inclination $\delta_{c l}$ is defined as follows (figure 5):

$$
-\frac{\pi}{2} \leq \alpha \leq \alpha_{\min } \Rightarrow \delta_{c l}=\delta_{c l, \max }
$$




$$
\begin{gathered}
\alpha_{\text {min }}<\alpha \leq 0 \quad \Rightarrow \delta_{c l}=1-\frac{\left(1-\delta_{c l, \text { max }}\right)}{\alpha_{\text {min }}} \cdot \alpha \\
0<\alpha \leq \alpha_{\text {max }} \quad \Rightarrow \delta_{c l}=1-\frac{\left(1-\delta_{c l, \text { min }}\right)}{\alpha_{\text {max }}} \cdot \alpha \\
\alpha_{\text {max }}<\alpha \leq \frac{\pi}{2} \quad \Rightarrow \delta_{c l}=\delta_{c l, \text { max }}
\end{gathered}
$$

In the case of a horizontally aligned path the correction factor is set to 1 , i.e. there is no correction. Downward aligned paths increase virtually the calculated cross-sectional area of cut and upward directed paths decrease it.

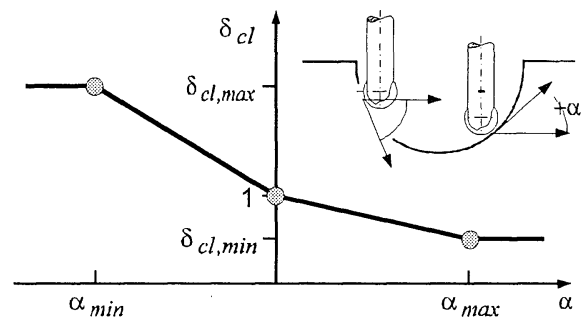

Figure 5. Definition of the correction factor for path inclination

\section{2..4 Aspects of machining dynamics}

In order to achieve the calculated feed-rate at the appropriate position it is necessary to take into account the dynamic capabilities of the machine (figure 6). In this case the deceleration length must be calculated and the new feed-rate value must be added to the NCfile at the correct position. The deceleration length is defined by:

$$
l_{d e c}=\frac{v_{f, a c t}{ }^{2}-v_{f, n e w}{ }^{2}}{2 \cdot a_{m}}+D
$$

with $a_{m}$ equal to the maximum deceleration of the machine. The first term in this expression is the real deceleration length caused by the limited dynamic capabilities of the machine, and the value $D$ (cutter diameter) is used as a safety parameter. It is used to ensure that the calculated optimal feed-rate is reached before the volume to be cut exceeds a given value.

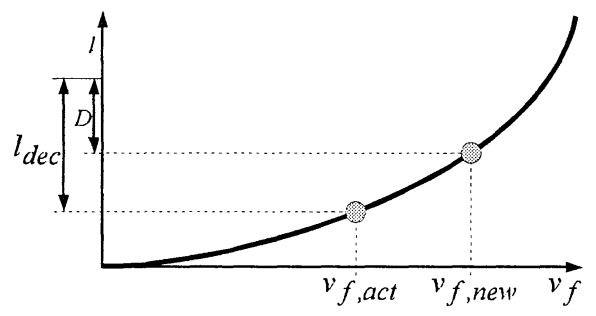

Figure 6. Calculation of the deceleration length

\section{THE SIMULATION KERNEL}

A method for calculating the actual surface geometry of the workpiece at any given point in the milling process and one which is not influenced by the size of the NC-file has been 
developed. The original idea is very simple and several modifications of this idea have been described before $[7,11]$ :

Let $M$ be a set of line segments in $3 \mathrm{~d}$ space defining the path of the reference point of a given cutter. Further, let $f$ be a function that represents the cutter. $f$ maps a symmetric region around the origin of a $2 \mathrm{~d}$ coordinate system into negative real numbers. Typical examples are flat-end cutters,

$$
f(x, y):=-r, x, y \in D(f):=\left\{(x, y) \mid x^{2}+y^{2} \leq r^{2}\right\},
$$

ball-end cutters

$$
f(x, y):=-\sqrt{r^{2}-x^{2}-y^{2}}, x, y \in D(f):=\left\{(x, y) \mid x^{2}+y^{2} \leq r^{2}\right\},
$$

and torus-end cutters

$$
f(x, y):=\left\{\begin{array}{c}
-r, x, y \in D(f):=\left\{(x, y) \mid x^{2}+y^{2} \leq(r-e)^{2}\right\} \\
-r+e-\sqrt{e^{2}-(r-x)^{2}-(r-y)^{2}}, x, y \in D(f):=\left\{(x, y) \mid \begin{array}{l}
x^{2}+y^{2}>(r-e)^{2} \\
x^{2}+y^{2} \leq r^{2}
\end{array}\right.
\end{array}\right\}
$$

The real number $r$ is a value for the size of the cutter. In both cases the origin is used as a reference point. The aim is to calculate the surface which is cut into the solid material when the reference point of the cutter is moved along the path $M$. First we assume the block of raw material to be a parallel-piped rectangle with external coordinates $\mathrm{x}_{\min }, \mathrm{x}_{\max }, \mathrm{y}_{\min }, \mathrm{y}_{\max }, \mathrm{z}_{\min }$ and $Z_{\max }$ in a suitable coordinate system. The surface can be described as the minimum of all points on the cutter surface at every cutter location along the path which additionally have to be clipped against the block of material,

$$
\begin{aligned}
& F(x, y):=\min \left\{z_{\max }, \min \left\{z \mid z:=s\left(x^{\prime}, y^{\prime}\right)+f\left(x-x^{\prime}, y-y^{\prime}\right),\right.\right. \\
& \left.\left.\left(x-x^{\prime}, y-y^{\prime}\right) \in D(f), z \geq z_{\min },\left(x^{\prime}, y^{\prime}, s\left(x^{\prime}, y^{\prime}\right)\right) \in M\right\}\right\}
\end{aligned}
$$

The exact calculation of $F(x, y)$ is time consuming, complicated and unstable due to the complex, detailed geometry of the resulting surface. An exact approach has been implemented by Kawashima [4]. An improvement on this approach has been made by Hui [3]. He avoids analytic calculations of the volumes of the chips swept out by the cutter. The main idea is to scan-convert the cutter path as well as the surface of the cutter. The scan-converted cutter is placed at each sampling point along the cutter path and the $\mathrm{z}$-value of the sampling grid is decreased according to the respective values for the scan-converted cutter surface.

The solution we have chosen is a discrete approach similar to the methods used by Hui [3] and Yazar [13]. In contrast to other approaches we improved the method in order to reduce the time and memory requirements of other straightforward implementations. The fundamental data structure of the model on which our algorithm operates contains the minimum and maximum $\mathrm{x}-, \mathrm{y}$ - and $\mathrm{z}$-coordinates of the material block on which the process is simulated. Furthermore, the data structure describes the resolution of the sampling grid in dots per millimeter. The z-heights are defined as having a resolution that is 256 times the resolution of the grid, in order to avoid staircase effects, and are represented by integers. The complete algorithm proceeds somewhat as follows.

scan-convert the surface of the cutter;

for the final grid point $q$ of the first segment do:

for all grid points $f$ of the scan-converted cutter within the material block do:

Cutpoint $(q+f, z)$, with $z=z(q)+z(f)$ the height value at $q+f$;

for all path segments do:

calculate the scan-converted cutting edge of the cutter;

for all grid points $r$ of the scan-converted path segment do:

for all grid points $s$ of the cutting edge placed at $r$ and lying within the material block do: 
Cutpoint $(s+r, z)$, with $z=z(s)+z(r)$ the height value at $s+r$;

for the final grid point $p$ of the path segment do:

for all grid points $f$ of the scan-converted cutter within the material block do:

Cutpoint $(p+f, z)$, with $z=z(p)+z(f)$ the height value at $p+f$;

The developed algorithm and its implementation is specified in [8].

\section{3..1 Calculation of the Cutting Edge of the Cutter}

The cutting edge of a cutter for a given path segment is the set of points on the surface of the cutter having a tangent parallel to the path segment. In order to decrease the simulation time, the reduction of cutting operations at each sampling point along the cutter path is achieved by using the cutting edge instead of the complete cutter surface. Figure 7 shows the cutting surface of a torus-end cutter for different directions. Let $\mathbf{I}$ be the length of a motion path in $\mathrm{mm}, \mathbf{d}$ the resolution of the sampling grid in $d o t s / \mathrm{mm}$ and $\mathbf{r}$ the radius of the cutter in $\mathrm{mm}$. The number of cutting operations $(\mathbf{N})$, without optimization, can be calculated as follows:

$$
\mathrm{N}(1, \mathrm{r}, \mathrm{d})=1 * \mathrm{~d} *(\mathrm{r} * \mathrm{~d})^{2} * \pi=1 * \mathrm{r}^{2} * \pi * \mathrm{~d}^{3} .
$$

Figure 7 shows the cutting edge optimization for different directions when using a torus cutter. The number of cutting operations (Nce), when using the cutting edge, can be expressed as:

$$
\operatorname{Nce}(1, \mathrm{r}, \mathrm{d}) \leq 1 * \mathrm{~d} * 2 * \mathrm{r} * \mathrm{~d} * \pi=1 * 2 * \mathrm{r} * \pi * \mathrm{~d}^{2}
$$
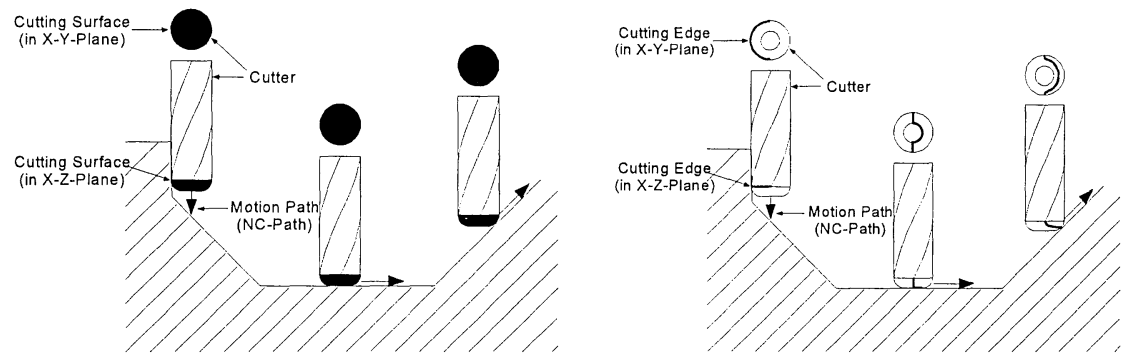

Figure 7. Cutting Surface and Cutting Edge for a torus cutter

Taking into account the main influence of $\mathbf{d}$ on the quality of the simulated surface and the simulation time, the simulation speed can be increased significantly by using the cutting edge. The points in figure 8 can be calculated as follows:

$$
\begin{aligned}
& x=-\sin (\theta+\phi) \cdot\left(r+(R-r)\left(1-\cos \left(\arctan \left(\frac{\left|\overrightarrow{v_{z}}\right|}{\sin \left(\theta-\frac{\pi}{2}\right) \cdot\left|\overrightarrow{v_{x}}+\overrightarrow{v_{y}}\right|}\right)\right)\right)\right. \\
& \left.\left.y=\cos (\theta+\phi) \cdot(r+(R-r))\left(1-\cos \left(\arctan \left(\frac{\left|\overrightarrow{v_{z}}\right|}{\sin \left(\theta-\frac{\pi}{2}\right) \cdot\left|\overrightarrow{v_{x}}+\vec{v}_{y}\right|}\right)\right)\right)\right)\right)
\end{aligned}
$$




\section{THE ADAPTIVE SIMULATION SYSTEM}

The conditions for the optimized feed-rate at each position on the NC-path is a user-given set of parameters [7]. These parameters are necessary for the analysis of the current engagement conditions and influence the feed-rates calculated by the simulation system. In the actual off-line simulation this set of parameters is fixed for one step in the milling process. The values for the parameters are set by experts. Figure 9 shows the simulation-based optimized process chain, going from the given NC-paths of a CAM-system to the milling process.

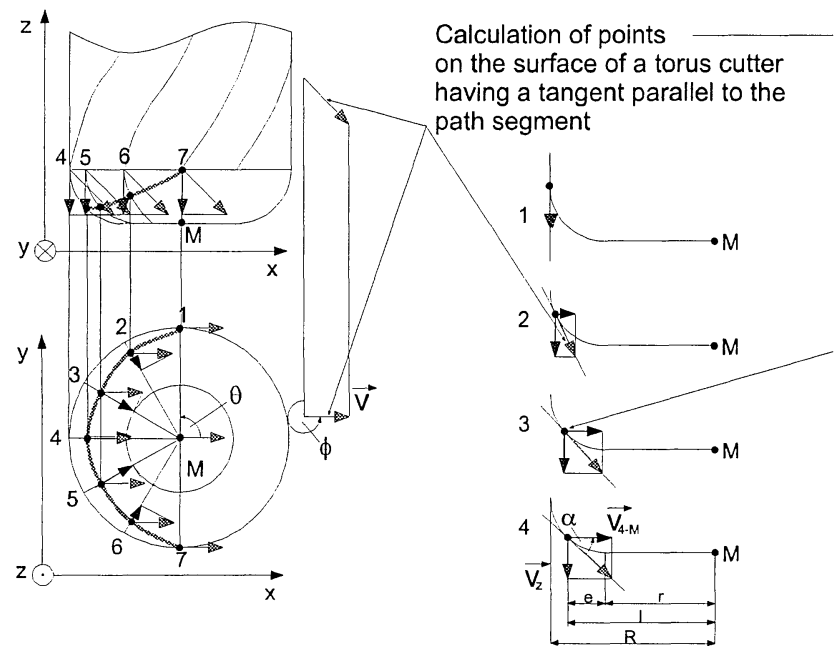

Figure 8. Calculation of the cutting edge for a given path segment

The milling simulation input is a file containing NC-paths and the information about the workpiece dimensions as described above. The output is a new file with optimized NC-paths which describe the same paths as the Input-file but with optimized feed-rates. The quality of the output depends on the simulation parameters chosen. The aim of the adaptive simulation system in figure 10 is to selflearn these parameters online during the milling process.

The simulation time takes only $10-25 \%$ of the real milling process on a Pentium 133 . This is the condition for an adaptive system which compares the recorded cutting-forces with the feed-rates calculated by the milling simulation. In the off-line optimization system of figure 9 the complete NC-paths are simulated before the milling process is started. The adaptive simulation system starts to optimize just a small part of the complete NC-paths with an initial parameter set for the simulation (1). After a selected length of NC-paths is processed, the milling process starts with these optimized paths. Simulation (1) is synchronised to the milling process in order to keep a constant distance of optimized milling paths as a buffer. Simulation (1) and (2) operate in the same way, but simulation (2) always operates at the same position along the path covered by the moving milling machine as the real milling process does. This is necessary for this system, because the real cutting forces should be compared with the calculated cutting forces of the simulation in order to adapt the internal simulation parameters online. After a set time the system adapts the simulation parameters of simulation (1) and (2) based on the results of the comparison undertaken. With this new configuration a second iteration of simulation, milling and comparison starts on the next part of the NC-path. In order to keep the parameters constant until the end of the NC-file the 
modification rate decreases from iteration to iteration. This is comparable to the idea of simulated annealing.

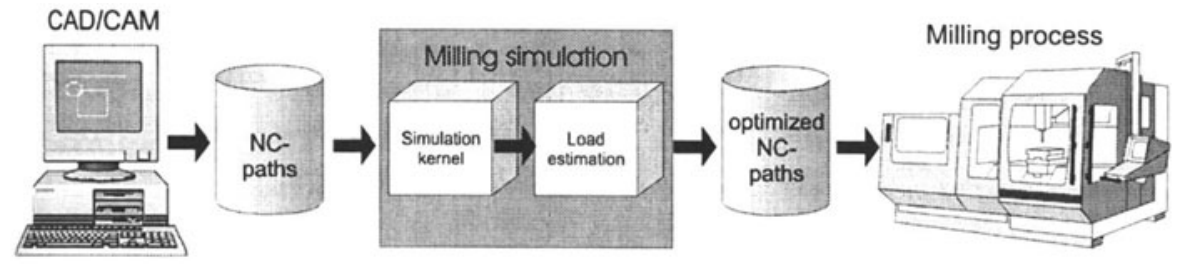

Figure 9. Part of the simulation based optimized process chain

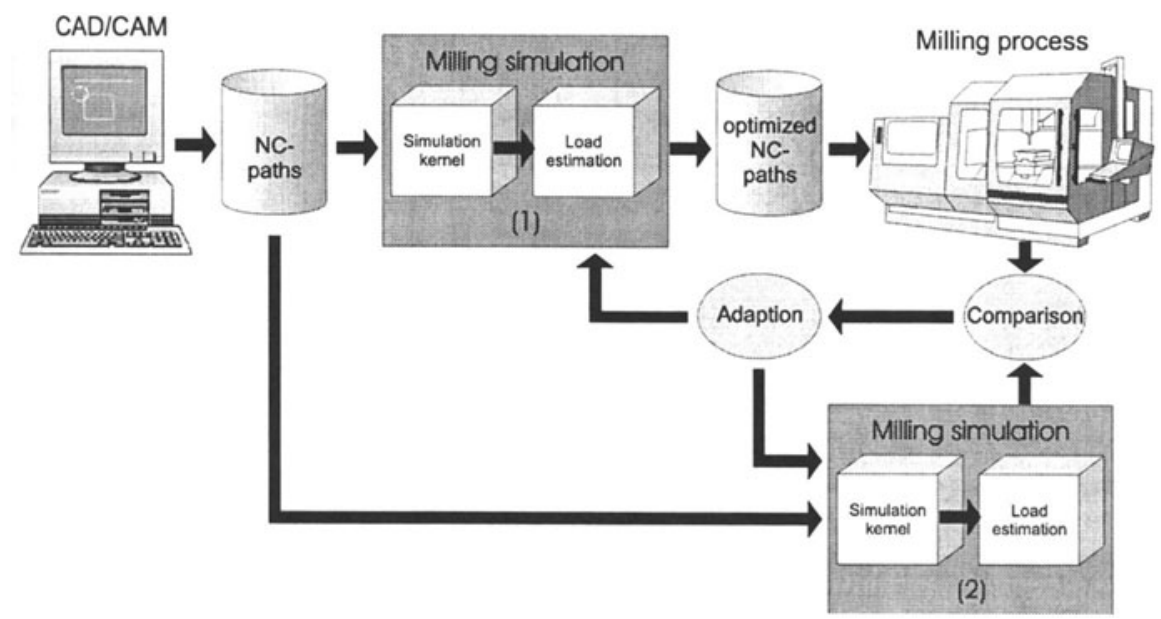

Figure 10. Adaptive Simulation System

Milling experiments in which typical geometries for the die- and mold-making industry are used show that the simulation is flexible enough to represent the real milling process closely enough.

Current work in this field of adaptive simulation is to work out strategies which adapt optimal parameters online. These resulting parameters should fit the given tool and workpiece combination and make it possible to start with an optimal set of parameters for the next step in the milling process. 

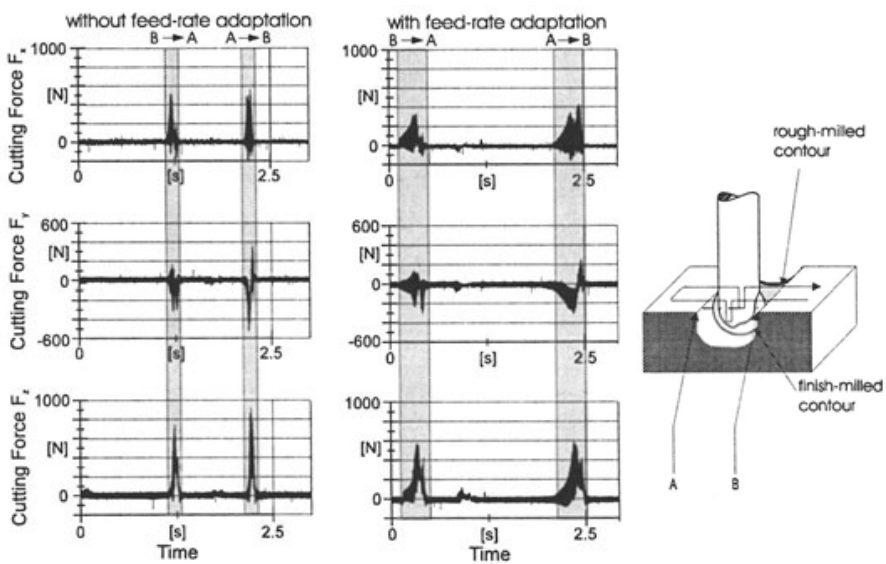

Figure 11. Measured cutting forces while milling with and without feed-rate adjustment

\section{APPLICATION EXAMPLE}

In order to show the capability of the milling simulation and optimization of the feed-rate which have been developed, a part with a typical geometry has been milled. The first step in machining is the rough milling by an $8-\mathrm{mm}$-diameter ball-end cutter using pocket-milling cycles. The next step is the semi-finish milling using a 6-mm-diameter ball-end cutter. The paths for this machining stage are generated in a rectilinear shape. A problem often arising while semi-finishing this part is the low process reliability, causing tool damages. While milling this part the cutting forces are recorded. As can be seen in figure 11, the volume in a narrow area of the part, i.e. an area that remains mostly unmachined by the $8 \mathrm{~mm}$ cutter, causes high tool loads that affect the $6 \mathrm{~mm}$ cutter (left).

In order to show the differences between the optimized and non-optimized process the cutting forces were recorded. Figure 11 shows these forces. The results of feed-rate adjustment are relatively stable tool loads without high cutting-force peaks resulting in stable cutting conditions and a satisfactory process reliability.

\section{REFERENCES}

1. Altintas, Y., (1996), "A General Mechanics and Dynamics Model for Helical End Mills", Annals of the CIRP, Vol. 45/1, pp. 59-64.

2. Bieker, R., (1991), "NC-Fräsen von Stahlhohlformen", VDI-Verlag, Düsseldorf.

3. Hui, K.C., (1994), "Solid Sweeping in Image Space-Application in NC-Simulation", The Visual Computer, Vol. 10, pp. 306-316.

4. Kawashima, Y., Itoh, K., et. al., (1991), "A flexible Quantitative Method for NC-Machining Verification Using a Space-Division based Solid Model", The Visual Computer, Vol. 7, pp. 149-157.

5. Spiewak, S., (1995), "An Improved Model of the Chip Thickness in Milling", Annals of the CIRP, Vol. 44/1, pp. 39-42.

6. Weinert, K. and Enselmann, A., (1995), "NC-Functions for HSC-Milling in the Field of Tool- and Mold-making", Flexible Automation and Intelligent Manufacturing (FAIM), Begell House inc., New York, pp. 957-967.

7. Weinert, K. and Enselmann, A., (1996), „A Model for Computer-Based Contour-Fault Prediction and Compensation when Milling Sculptured Surfaces", Flexible Automation and Intelligent Manufacturing (FAIM), Begell House inc., New York, pp. 915-928. 
8. Weinert, K., Müller, H. and Friedhoff, J., (1997), "Efficient Discrete Simulation of 3-Axis-Milling for Sculptured Surfaces", Production Engineering, Vol. III/2.

9. Weinert, K., Enselmann, A.. and Albersmann, F., (1997), "Feed-Rate Adaption, Contour-Fault Prediction and Compensation for Optimisation of the HSC-Milling Process," Proceedings of the European Conference on Integration in Manufacturing, Technical University of Dresden, Germany, pp. 301-312.

10. Werner, A., (1992), "Prozeßauslegung und Prozeßsicherheit beim Einsatz von schlanken Schaftfräsern", Dissertation, WZL, RWTH Aachen, Germany.

11. Yang, B. and Menq, C., (1993), "Compensation for Form Error of End-Milled Sculptured Surfaces Using Discrete Measurement Data", Int. J. Mach. Tools Manufact., Vol. 33, pp.725-740.

12. Yang, M. and Sim, C., (1993), "Reduction of Machining Errors by Adjustment of Feed-Rates in the Ball-End Milling-Process", Int. J. Prod. Res., Vol. 31, pp. 665-689.

13. Yazar, Z., Koch, K., et. al., (1994), "Feed-Rate Optimization Based on Cutting Force Calculations in 3-Axis Milling of Dies and Molds with Sculptured Surfaces", Int. J. Mach. Tools. Manufact., Vol. 34, pp. 365-377. 